\title{
First-principles study of the structural energetics of PdTi and PtTi
}

\author{
Xiangyang Huang, Karin M. Rabe and Graeme J. Ackland \\ Department of Physics and Astronomy, Rutgers University, Piscataway, New Jersey 08854-8019
}

(Dated: November 4, 2018)

\begin{abstract}
The structural energetics of PdTi and PtTi have been studied using first-principles densityfunctional theory with pseudopotentials and a plane-wave basis. We predict that in both materials, the experimentally reported orthorhombic $B 19$ phase will undergo a low-temperature phase transition to a monoclinic $B 19^{\prime}$ ground state. Within a soft-mode framework, we relate the $B 19$ structure to the cubic $B 2$ structure, observed at high temperature, and the $B 19^{\prime}$ structure to $B 19$ via phonon modes strongly coupled to strain. In contrast to NiTi, the B19 structure is extremely close to hcp. We draw on the analogy to the bcc-hcp transition to suggest likely transition mechanisms in the present case.
\end{abstract}

PACS numbers:

\section{INTRODUCTION}

Shape memory alloys (SMA) have attracted a great deal of attention due to their important technological applications, including mechanical actuator devices and medical stents. The shape memory effect also gives rise to superelasticity, which finds applications in stents and spectacle frames.

The shape memory effect is related to a reversible martensitic (diffusionless) phase transformation. It has been shown that the martensitic transformation can be induced by applied fields, temperature or both, and the mechanical properties of materials, therefore, can be controlled accordingly. In many systems, including those discussed in the present work, alloying can dramatically change the properties and transition temperatures of the materials, reflecting the importance of electronic features, specifically Fermi surface effects, in the structural energetics of SMA.

There are several complementary approaches to modelling of the shape memory effect. Continuum modelling allows investigation of the microstructural behavior, specifically martensitic twins, at the relevant long length scales. Material-specific behavior is incorporated through an empirical functional for the free energy in terms of strain and a twin boundary energy to set the length scale. In atomistic models, the individual atoms are considered explicitly and their interaction given by an interatomic potential, which may be determined empirically, from first-principles density-functional-theory (DFT) calculations, or a combination of the two. Crystal symmetry and defect energies emerge from this approach, which gives microstructures with both natural length scales (from defect energies) and time scales (since the atoms have definite Newtonian forces and masses). However, in atomistic models, the electronic degrees of freedom do not appear explicitly. First principles DFT methods are so computationally intensive that direct studies of microstructural behavior are impossible, but they are valuable both for obtaining quantitative atomiclevel information regarding energies, forces and stresses independent of empirical input, and for understand- ing the electronic origin of this behavior. Thus, firstprinciples investigation of the energetic instability of the high-temperature structure towards the low-symmetry martensitic structure is in itself quite illuminating. The resulting information can then also be used as inputs to atomistic [i] and continuum modelling of shape memory behavior.

Typically, martensitic transformations are described using the strain as an order parameter, the classic example being the Bain bcc-fcc transformation of iron. However, there is an alternative approach appropriate for cases where the strain degrees of freedom are coupled to atomic degrees of freedom (phonons). Following the softmode theory of structural transitions, [2] we start from a high-symmetry reference structure (here $B 2$ ) and freeze in unstable phonons of this structure, with corresponding lattice relaxation, to produce the ground-state structure. The symmetry of the phonons determines the symmetry of the low temperature structure. This approach has been successfully used in the study of minerals 3 , 何, 51 and ferroelectric materials [6, 7, 8, 9] and has been extended to shape memory alloys in our previous study of $\mathrm{NiTi} 10]$.

Closely related to NiTi, PdTi and PtTi are shape memory materials with reasonable structural simplicity and extraordinary mechanical behavior. They undergo a martensitic transformation at tunable temperatures: PdTi transforms at $810 \mathrm{~K}$, but this can be reduced to $410 \mathrm{~K}$ with $8 \%$ substitution of $\mathrm{Cr}$ for $\mathrm{Pd}[11$. The high-temperature "austenitic" phase has a simple cubic $B 2$ structure (space group $P m 3 \bar{m}$ ), while the ambient temperature "martensitic" phase has been reported as the orthorhombic B19 structure 12, 13 (space group: Pmma). Previous first-principles studies in PdTi and PtTi 14, 15, 16] have shown that the observed electronic and elastic properties of the $B 19$ structure are well reproduced by density-functional theory calculations assuming the experimentally determined structure.

In this paper, we investigate the structural energetics of PdTi and PtTi from first-principles calculations of phonon frequencies as well as total energies. This allows us to examine local as well as global stability and to in- 
vestigate transition mechanisms, drawing on the strong analogy between the $B 2-B 19$ and bcc-hcp transformations and showing that coupling of unstable modes to the strain is a crucial aspect of the structural energetics. In Sec. II, we describe the first-principles calculations. In Sec. III, we present and discuss the results for the phonon dispersion of PdTi and PtTi in the B2 structure and for the relaxed structures in which unstable modes and strains are coupled, yielding a low-symmetry ground state. In addition, we present results of calculations of the electronic structure, identifying and discussing features that affect the relative stability of the phases. Sec. $\mathrm{V}$ concludes the paper.

\section{CALCULATIONS}

First-principles total energy calculations were carried out within density-functional theory with a plane-wave pseudopotential approach. The calculations were performed with the Vienna ab-initio Simulations Package 17 . 18], using the Perdew-Zunger 19 parametrization of the local-density approximation (LDA). Vanderbilt ultrasoft pseudopotentials [20] were used. Our pseudopotentials include nonlinear core corrections and for $\mathrm{Ti}$, we treated the occupied $3 p$ levels as valence. The electronic wave functions were represented in a plane-wave basis set with a kinetic energy cutoff of $278 \mathrm{eV}$. The Brillouin zone (BZ) integrations were carried out by the Hermite-Gaussian smearing technique 21] with the smearing parameter of $0.1 \mathrm{eV}$. The unit cells contain two atoms in the cubic $B 2$ structure and four atoms in the orthorhombic $B 19$ and monoclinic $B 19^{\prime}$ structures. The calculations were performed with a $16 \times 16 \times 16$ Monkhorst-Pack $(\mathrm{MP}) k$-point mesh for the cubic $B 2$ structure and a $12 \times 12 \times 16 \mathrm{MP}$ $k$-point mesh for both orthorhombic $B 19$ and monoclinic $B 19^{\prime}$ structures (space group: $P 2_{1} / m$ ), corresponding to $120 k$ points in the $\frac{1}{48}$ irreducible $\mathrm{BZ}$ of the simple cubic cell, $288 k$ points in the $\frac{1}{8}$ irreducible BZ of the orthorhombic cell and $576 k$ points in the $\frac{1}{4}$ irreducible BZ of the monoclinic cell. This choice of parameters converges the total energy to within $1 \mathrm{meV} /$ atom. The density of states (DOS) for the $B 19$ and $B 19^{\prime}$ structures were calculated using the tetrahedron method with Blöchl corrections 22]. The BZ's for the orthorhombic $B 19$ and monoclinic $B 19^{\prime}$ structures are different. To compare the band structure of the two structures, we label the $B 19$ band structure by regarding the $B 19$ structure as a special case of $B 19^{\prime}$.

The phonon dispersion relations were obtained with the PWSCF and PHONON codes [23], using the PerdewZunger 19] parametrization of the LDA, as above. Ultrasoft pseudopotentials [20] for $\mathrm{Pd}, \mathrm{Pt}$ and $\mathrm{Ti}$ were generated according to a modified Rappe-Rabe-KaxirasJoannopoulos (RRKJ) scheme [24] with three Bessel functions 25. The electronic wave functions were represented in a plane-wave basis set with a kinetic energy cutoff of $408 \mathrm{eV}$. The augmentation charges were expanded up to $9000 \mathrm{eV}$. The Brillouin zone (BZ) integrations were carried out by the Hermite-Gaussian smearing technique 21] using a $56 k$-point mesh (corresponding to $12 \times 12 \times 12$ regular divisions along the $k_{x}, k_{y}$ and $k_{z}$ axes) in the $\frac{1}{48}$ irreducible wedge. The value of the smearing parameter was $\sigma=0.2 \mathrm{eV}$. These parameters yield phonon frequencies converged within $5 \mathrm{~cm}^{-1}$. The dynamical matrix was computed on a $6 \times 6 \times 6 q$-point mesh commensurate with the $k$-point mesh. The complete phonon dispersion relation was obtained through the computation of realspace interatomic force constants within the corresponding box 26].

The choice to use two different first-principles codes was dictated by the individual strengths of each. VASP has a highly efficient scheme for calculating total energies, forces, and stresses, and relaxing to the minimum energy structure, but does not have the density-functional perturbation theory capabilities of PWSCF/PHONON. Even with slightly different pseudopotentials and $k$-point sampling, the results of the two codes are quite compatible. For example, the difference between the computed lattice parameters for the $B 2$ structure of PdTi is less than $0.2 \%$, and for PtTi the difference is less than $0.1 \%$. Comparisons of normalized eigenvector components computed by VASP using the frozen phonon method and by PWSCF/PHONON also show good agreement, generally within $5 \%$.

\section{RESULTS}

For the cubic $B 2$ structure, our calculations yield the equilibrium lattice parameters of $3.112 \AA$ and $3.125 \AA$ for PdTi and PtTi respectively. For comparison, we also performed full-potential linearized-augmentedplane-wave calculations (FLAPW) within the LDA[27. The results are in excellent agreement with FLAPW results of $3.113 \AA$ (PdTi) and $3.127 \AA(\mathrm{PtTi})$ and in in good agreement with experimental values of $3.18 \AA$ and $3.17 \AA$.

The phonon dispersion relations along high symmetry lines, computed at the theoretical lattice parameters, are shown in Figure 11. The frequencies are obtained by taking the square root of the eigenvalues of the dynamical matrix 29. Imaginary frequencies, as plotted as negative, are obtained from negative eigenvalues of the dynamical matrix. Thus, the structure is dynamically unstable against distortions following the corresponding eigenvector. It should be noted that the phonon frequency $\omega$ is not the reciprocal of the period of oscillation of this mode (as measured in molecular dynamics) nor is it the energy difference between adjacent quantum levels (as measured in neutron scattering experiments). These three quantities are equal only for a stable harmonic crystal. In the materials studied here the unstable modes may be related to a soft mode[2] (as defined by MD or neutron scattering) at high temperature, and even the stable modes are expected to be significantly renormalized as a function of temperature by anharmonic effects. 

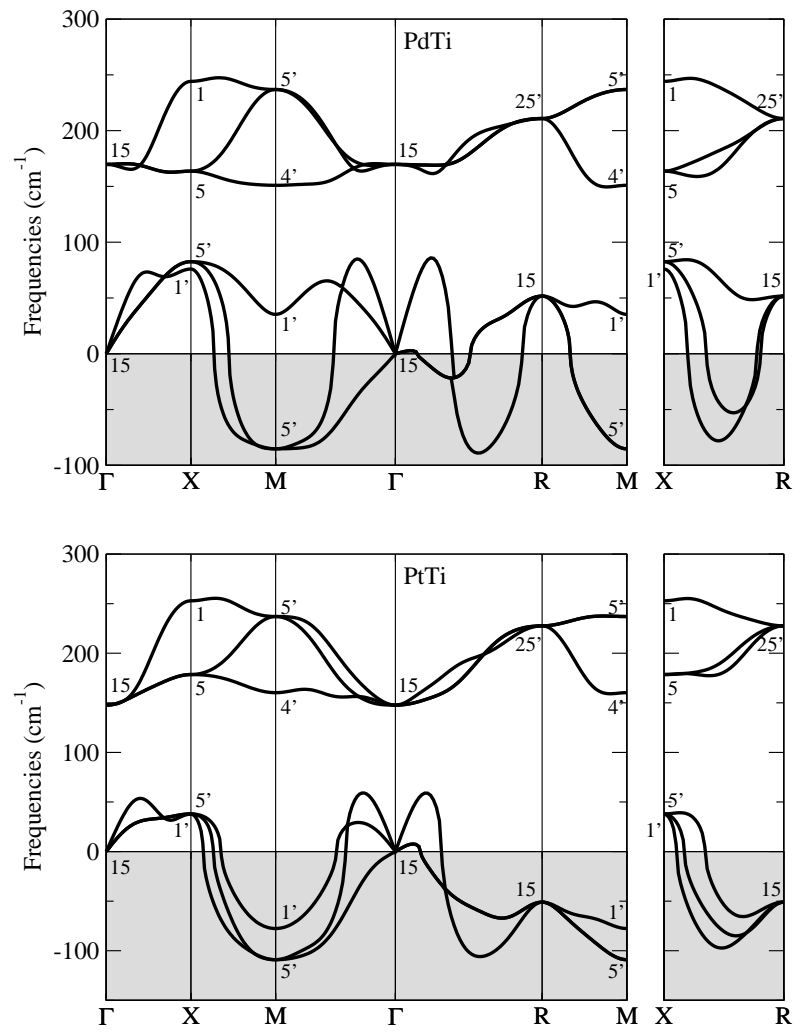

FIG. 1: Phonon dispersion relations for PdTi (upper) and PtTi (lower) in the $B 2$ structure calculated at the LDA equilibrium parameters $3.112 \AA$ and $3.125 \AA$ respectively. The negative slope of the acoustic $\Gamma-M$ branch corresponds to a pure elastic instability $\left(c^{\prime}=\frac{1}{2}\left(c_{11}-c_{12}\right)<0\right)$. Symmetry labels are assigned according to the conventions of Ref. 28 with $\mathrm{Pd} / \mathrm{Pt}$ at the origin. The imaginary frequencies of the unstable modes are plotted as negative values.

The dynamical matrices are related by mass factors to the force constant matrix: the second derivatives of the internal energy with respect to atomic displacements. The eigenmodes of the force constant matrix describe the potential energy landscape, and a negative eigenvalue indicates a static instability against a distortion following the corresponding eigenvector. While the actual normalized displacements of these eigenmodes are in general slightly different they carry the same symmetry labels as the eigenmodes of the dynamical matrix. Either choice is expected to serve as a useful pointer to a lower energy structure if the distorted structure obtained by "freezing in" an unstable mode is relaxed using first-principles forces and stresses, as we describe below.

The phonon dispersion relations shown in Figure 1 show instability of the $B 2$ structure similar to and even stronger than that of $\mathrm{NiTi} 110]$. There are large regions of reciprocal space where one, two or even three modes are unstable, with dominant instabilities at $M$ and along $\Gamma-R$. The phonon instability shows that the observed high-temperature $B 2$ phases of PdTi and PtTi are dynamically stabilized by anharmonic phonons, and should be characterized by large fluctuating local distortions. The calculated phonon dispersions are also reminiscent of those of unstable bcc materials such as $\mathrm{Zr}$ and $\mathrm{Ti}$, which undergo martensitic transformations to hcp or $\omega[30]$ (via the $M$ and $\Gamma-R$ bcc-phonon equivalents respectively) phases. The analogy based on the view of $B 19$ phases of PdTi and PtTi as chemically ordered hcp will be further strengthened below.

In the soft mode approach, we search for local energy minima by choosing an unstable mode of the high symmetry structure, freezing in the distortion with varying amplitude, and relaxing the resulting structure. In many cases, the mode with the largest negative eigenvalue will generate the lowest energy structure. However, this is by no means generally true, as the energy gain is determined not only by the curvature of the energy surface but by higher order terms as well as the strength of coupling to strain and other modes, both unstable and stable, of appropriate symmetry. Indeed, in PdTi this "most unstable" mode (i.e. largest negative eigenvalue) lies in the $\Gamma-R$ branch rather than at $M$. This mode is typically unstable in dynamically-stabilized bcc materials such as titanium and zirconium where it is associated with a phase transition to the ideal $\omega(C 32)$ phase. The fact that in the chemically ordered analog $(\mathrm{Pd} / \mathrm{Pt}) \mathrm{Ti}$, the observed phase transition is to $B 19$ rather than $C 32$ illustrates the importance of anharmonic effects and strain coupling in the energetics of these materials.

In the $B 2$ structure, a doubly-degenerate unstable $M_{5}^{\prime}$ zone-boundary mode implies a doubling of the unit cell to $\mathbf{a}=(001)_{b c c}, \mathbf{b}=(110)_{b c c}, \mathbf{c}=(1 \overline{1} 0)_{b c c}$. For the distortion to be frozen in, we choose the eigenvector that gives the space group Pmma of the B19 structure (Figure 2). The unit cell is orthorhombic, with lattice parameters $a, b$ and $c$, two Wyckoff positions occupied by Ti and $\mathrm{Pd} / \mathrm{Pt}$

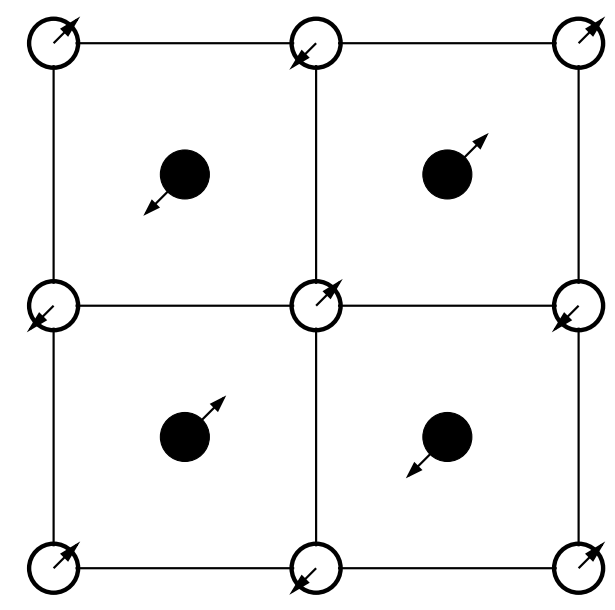

FIG. 2: Eigenmode of the doubly-degenerate $M_{5}^{\prime}$ unstable phonon in the $B 2$ structure. This mode generates the $B 19$ structure in PdTi and PtTi. The structure is shown projected along the $\hat{z}$ direction, with $\mathrm{Pd} / \mathrm{Pt}$ represented by filled circles and Ti by open circles. 
TABLE I: Computed structural parameters and total energies of PdTi and PtTi in the B19 and B19' structures, compared with the computed $L 1_{0}$ structure, the computed bco structure, the experimental $B 19$ structure, with previous calculations 16 . and with two special $B 19$ structures with $b / a$ and $c / a$ corresponding to ideal hcp and $B 2$ (bcc) structures. Volume is given in $\AA^{3} /$ formula unit, and energy in eV/atom. Wyckoff positions refer to space group $P 2_{1} / m\left(B 19^{\prime}\right)$.

\begin{tabular}{|c|c|c|c|c|c|c|c|c|c|c|}
\hline & Structure & Volume & $b / a$ & $c / a$ & $\gamma\left({ }^{\circ}\right)$ & Wyckoff position & $x$ & $y$ & $z$ & $E-E_{B 2}$ \\
\hline \multirow[t]{15}{*}{$\mathrm{PdTi}$} & $B 19$ & 29.63 & 1.753 & 1.634 & 90 & $\operatorname{Pd}(2 \mathrm{e})$ & 0 & 0.6866 & 0.25 & -0.0917 \\
\hline & & & & & & $\operatorname{Ti}(2 \mathrm{e})$ & 0.5 & 0.2008 & 0.25 & \\
\hline & $B 19^{\prime}$ & 29.64 & 1.758 & 1.633 & 93.39 & $\operatorname{Pd}(2 \mathrm{e})$ & 0.0114 & 0.6827 & 0.25 & -0.0924 \\
\hline & & & & & & $\operatorname{Ti}(2 \mathrm{e})$ & 0.4475 & 0.1964 & 0.25 & \\
\hline & $L 1_{0}$ & 29.32 & 1.000 & 1.375 & 90 & & & & & -0.0870 \\
\hline & bco & 30.16 & 1.837 & 1.579 & 105.79 & $\operatorname{Pd}(2 \mathrm{e})$ & 0.0882 & 0.6764 & 0.25 & -0.0573 \\
\hline & & & & & & $\operatorname{Ti}(2 \mathrm{e})$ & 0.3491 & 0.1983 & 0.25 & \\
\hline & $B 19^{a}$ & 31.33 & 1.74 & 1.62 & 90 & & & & & \\
\hline & $B 19^{b}$ & 31.74 & 1.75 & 1.64 & 90 & & & & & \\
\hline & $B 19^{c}$ & 30.3 & 1.72 & 1.62 & 90 & $\operatorname{Pd}(2 \mathrm{e})$ & 0 & 0.689 & 0.25 & -0.095 \\
\hline & & & & & & $\operatorname{Ti}(2 \mathrm{e})$ & 0.5 & 0.201 & 0.25 & \\
\hline & special $B 19$ (hcp) & & 1.732 & 1.633 & 90 & $\overline{P d}(2 \mathrm{e})$ & 0 & 0.6666 & 0.25 & \\
\hline & & & & & & $\operatorname{Ti}(2 \mathrm{e})$ & 0.5 & 0.1666 & 0.25 & \\
\hline & special $B 19(B 2)$ & & 1.414 & 1.414 & 90 & $\operatorname{Pd}(2 \mathrm{e})$ & 0 & 0.5 & 0.25 & \\
\hline & & & & & & $\operatorname{Ti}(2 \mathrm{e})$ & 0.5 & 0.0 & 0.25 & \\
\hline \multirow[t]{11}{*}{ PtTi } & $\overline{B 19}$ & 29.84 & 1.758 & 1.668 & 90 & $\mathrm{Pt}(2 \mathrm{e})$ & 0 & 0.6874 & 0.25 & -0.1512 \\
\hline & & & & & & $\operatorname{Ti}(2 \mathrm{e})$ & 0.5 & 0.1958 & 0.25 & \\
\hline & $B 19^{\prime}$ & 29.88 & 1.762 & 1.656 & 93.56 & $\operatorname{Pt}(2 \mathrm{e})$ & 0.0154 & 0.6841 & 0.25 & -0.1561 \\
\hline & & & & & & $\operatorname{Ti}(2 \mathrm{e})$ & 0.4455 & 0.1923 & 0.25 & \\
\hline & $L 1_{0}$ & 29.52 & 1.000 & 1.386 & 90 & & & & & -0.1397 \\
\hline & bco & 30.57 & 1.835 & 1.561 & 105.80 & $\operatorname{Pt}(2 \mathrm{e})$ & 0.0907 & 0.6816 & 0.25 & -0.1231 \\
\hline & & & & & & $\operatorname{Ti}(2 \mathrm{e})$ & 0.3502 & 0.2001 & 0.25 & \\
\hline & $B 19^{a}$ & 30.66 & 1.75 & 1.663 & 90 & & & & & \\
\hline & $B 19^{b}$ & 29.75 & 1.75 & 1.666 & 90 & & & & & \\
\hline & $B 19^{c}$ & 30.9 & 1.72 & 1.62 & 90 & $\operatorname{Pt}(2 e)$ & 0 & 0.688 & 0.25 & -0.155 \\
\hline & & & & & & $\operatorname{Ti}(2 \mathrm{e})$ & 0.5 & 0.197 & 0.25 & \\
\hline
\end{tabular}

${ }^{a} \mathrm{X}$-ray, from Ref. 12 .

${ }^{b} \mathrm{X}$-ray, from Ref. $\overline{13}$.

${ }^{c}$ previous calculation, from Ref. 16 .

yielding a total of two free internal parameters $v_{P d / P t}$ and $v_{T i}$. The unstable mode corresponds to a distortion with fixed $v_{P d / P t} / v_{T i}$ ratio. In $\mathrm{PdTi}$, the ratios for the phonon and unstable force constant eigenmode as computed by PWSCF (VASP) are 1.55 (1.48) and 1.75 (1.64), respectively. In $\mathrm{PtTi}$, the corresponding ratios are 1.25 (1.19) and 1.81 (1.62), respectively.

In Table If, we provide the calculated equilibrium lattice parameters of the $B 19$ structure, obtained by relaxing all five free structural parameters. The results are in good agreement with experiment, aside from the volume underestimate typical of LDA. The computed relaxed values of the ratio $v_{P d / P t} / v_{T i}$ are 1.29 for PdTi and 1.16 for PtTi. The latter is quite close to the VASP phonon result, while the former is significantly lower. To understand this result more fully, we separate the coupling to strain from that to the second (stable) mode of the same symmetry by relaxing the structure to $B 19$ with the lattice held fixed. The resulting ratios $v_{P d / P t} / v_{T i}$ are 1.51 for PdTi and 1.41 for PtTi. Thus both couplings are significant in both systems, though the effects fortutiously nearly cancel in PtTi. This behavior is compatible with continuum models 31.

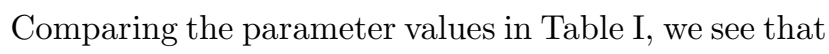
the relaxed $B 19$ structure is close to the ideal hcp structure that would be obtained from packing monodisperse hard spheres, with parameters given in the last line of the Table I. The lower symmetry arises from the "decoration" of the close packed plane with two different atomic species, which cannot preserve symmetry and maximise unlike near neighbors 32. This confirms that the phase transition is best thought of as a binary equivalent of bcc-hcp, not simply as a distortion of the $B 2$ structure. There is an interesting contrast with NiTi here: the experimentally reported ground state NiTi $B 19^{\prime}$ phase has $b / a=1.603$ and $c / a=1.417$ [33], far from hcp.

In the soft-mode approach, there is no guarantee that the energy-minimizing freezing-in of one unstable mode will stablize the other unstable modes of the highsymmetry structure. In the present case, the undistorted doubled unit cell contains two sets of $(110)_{b c c}$ planes each of which is unstable to strain-coupled shuffling at all $q$ points. The simplest such mode in the $B 19$ structure is $\Gamma_{4}$, which lowers the symmetry to monoclinic $P 2_{1} / \mathrm{m}$. The resulting $B 19^{\prime}$ structure has three additional free parameters: the monoclinic angle $\gamma$ and two internal pa- 

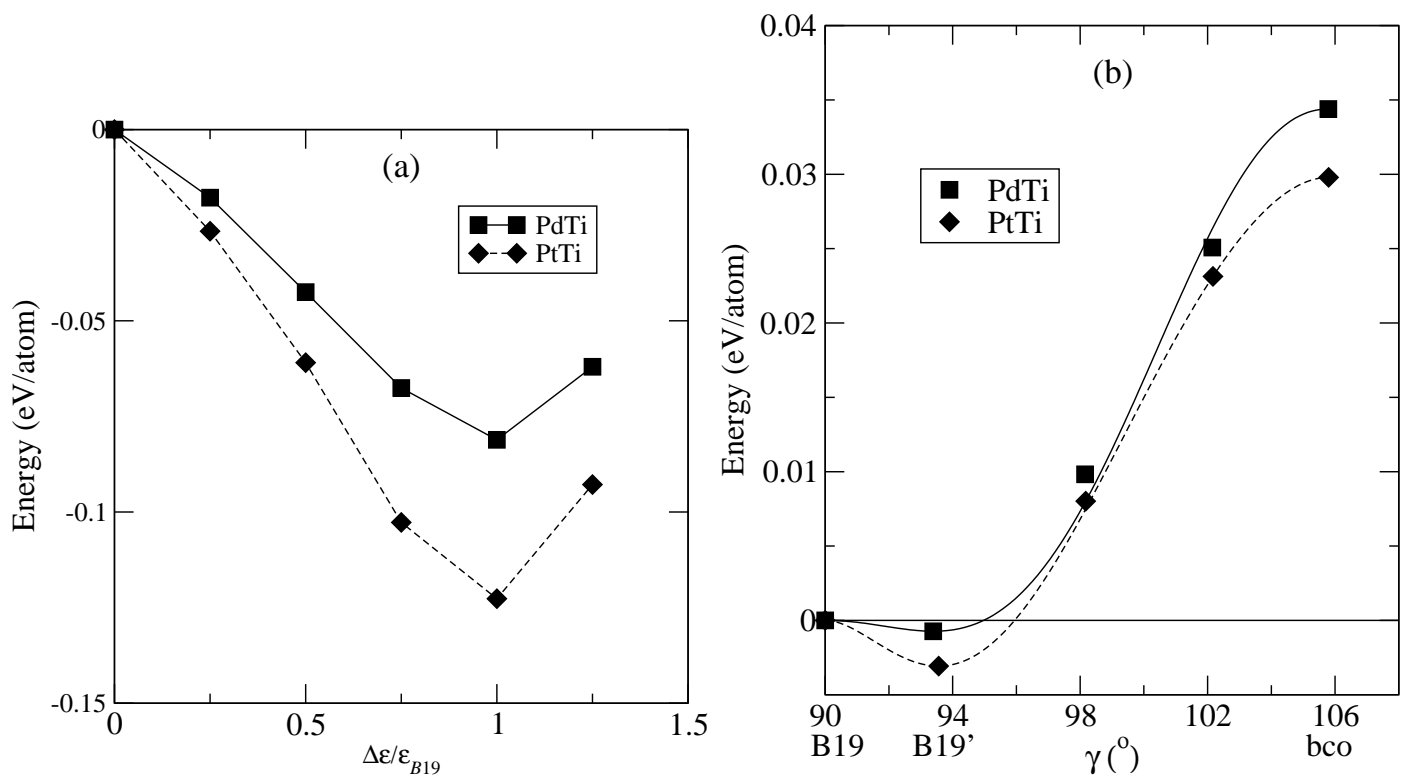

FIG. 3: (a) Total energies as a function of uniaxial strain with the internal coordinates being fully relaxed. The lattice parameters for $\Delta \varepsilon / \varepsilon_{B 19}=0,1$ are taken from $B 2$ and $B 19$ structures, while others were fixed by interpolation. The "B2" structure here corresponds to freezing in the $M$-point phonon, and lies $10.5 \mathrm{meV} /$ atom $(\mathrm{PdTi})$ or $30.2 \mathrm{meV} / \mathrm{atom}(\mathrm{PtTi})$ below the fully symmetric $(v=0)$ structures. (b)Total energies as a function of monoclinic angle. For $B 19, B 19^{\prime}$ and bco all degrees of freedom are relaxed. For intermediate angles the lattice parameters $a, b, c$ were fixed by interpolation, while the internal coordinates $u, v$ were fully relaxed. $u$ and $v$ vary almost linearly with $\gamma$. Symbols indicate calculated values, curve is a polynomial fit with $d E / d \gamma=0$ enforced where required by symmetry.

TABLE II: Optical phonon frequencies at $\Gamma$ for PdTi and PtTi in the $B 19$ and bco structures by DFPT. The lowest frequency mode couples to the strain in the $B 19-B 19^{\prime}$ transition.

\begin{tabular}{ccc}
\hline \hline Alloy & structure & $\omega\left(\mathrm{cm}^{-1}\right)$ \\
\hline PdTi & $B 19$ & $73,90,101,139,159,184,199,231,251$ \\
& bco & $94,138,146,148,164,198,200,230,256$ \\
PtTi & B19 & $68,87,98,123,165,185,196,238,260$ \\
& bco & $119,171,172,173,185,210,225,247,284$ \\
\hline \hline
\end{tabular}

TABLE III: Calculated optical phonon frequencies and ratio of atomic displacements of $\Gamma_{4}^{\prime}$ mode of PdTi and PtTi at relaxed $B 19$ structure from force constant matrix eigenvectors (i.e. ignoring masses). The values in italic are the modes leading to $B 19^{\prime}$ structure and are compared with values in parentheses which are taken from relaxed $B 19^{\prime}$ structure. Frequencies here differ slightly from Table II because they are calculated from finite displacements 34] using VASP rather than DFPT.

\begin{tabular}{llc}
\hline \hline Alloy & Frequencies & $u_{P d / P t} / u_{T i}$ \\
\hline PdTi & $74,103,159$ & $-0.280(-0.217), 3.5684,-1.002$ \\
PtTi & $69,106,161$ & $-0.321(-0.283), 3.1159,-1.05$ \\
\hline \hline
\end{tabular}

rameters $u_{T i}$ and $u_{P d}$. These values are given for the relaxed $B 19^{\prime}$ structure in Table If and compared with the normalized eigenvector displacements in Table III. However, it is important to note that the computed $\Gamma$ phonon modes in the $B 19$ structures are in fact all stable (Table [1]). The lowering of energy by distortion to $B 19^{\prime}$ cannot be obtained by a pure $\Gamma_{4}$ phonon distortion, but only if the strain is allowed to relax simulataneously (Table $\mathbb{1}$ ). This may be the reason that in a previous calculation [16], B19 was reported to be the minimum energy structure.

The relative energies of the various relaxed structures are given in Table I. The B19 total energy is lower than B2. A simple estimate of the transition temperature is given by $\Delta E=k T_{c}$ which suggest $T_{c}$ of $1050 \mathrm{~K}(\mathrm{PdTi})$ and $1755 \mathrm{~K}(\mathrm{PtTi})$. These rough values are significantly larger than the experimental data for the hysteretical transition region 13 , 35, 36 (approximately 800K and $1400 \mathrm{~K}$ respectively), but show the correct material trend. For neither system has a $B 19^{\prime}$ phase yet been observed experimentally. The small computed energy differences between $B 19^{\prime}$ and $B 19^{\prime}$, translated into temperature, are $28 \mathrm{~K}$ and $39 \mathrm{~K}$ for PdTi and PtTi, respectively. This suggests that the transition to the $B 19^{\prime}$ phase should occur at temperatures well below those at which the experiments were performed, so that our results are fully consistent with the available experimental work.

The binary-hcp phase interpretation also suggests that we should examine the binary-fcc equivalent, which is the $L 1_{0}$ phase. $L 1_{0}$ is accessible from $B 2$ by a simple (001) shear and has lower energy 14, however we find that $L 1_{0}$ has slightly higher energy than $B 19$. We consider one further structure: at the special $B 19^{\prime}$ values 

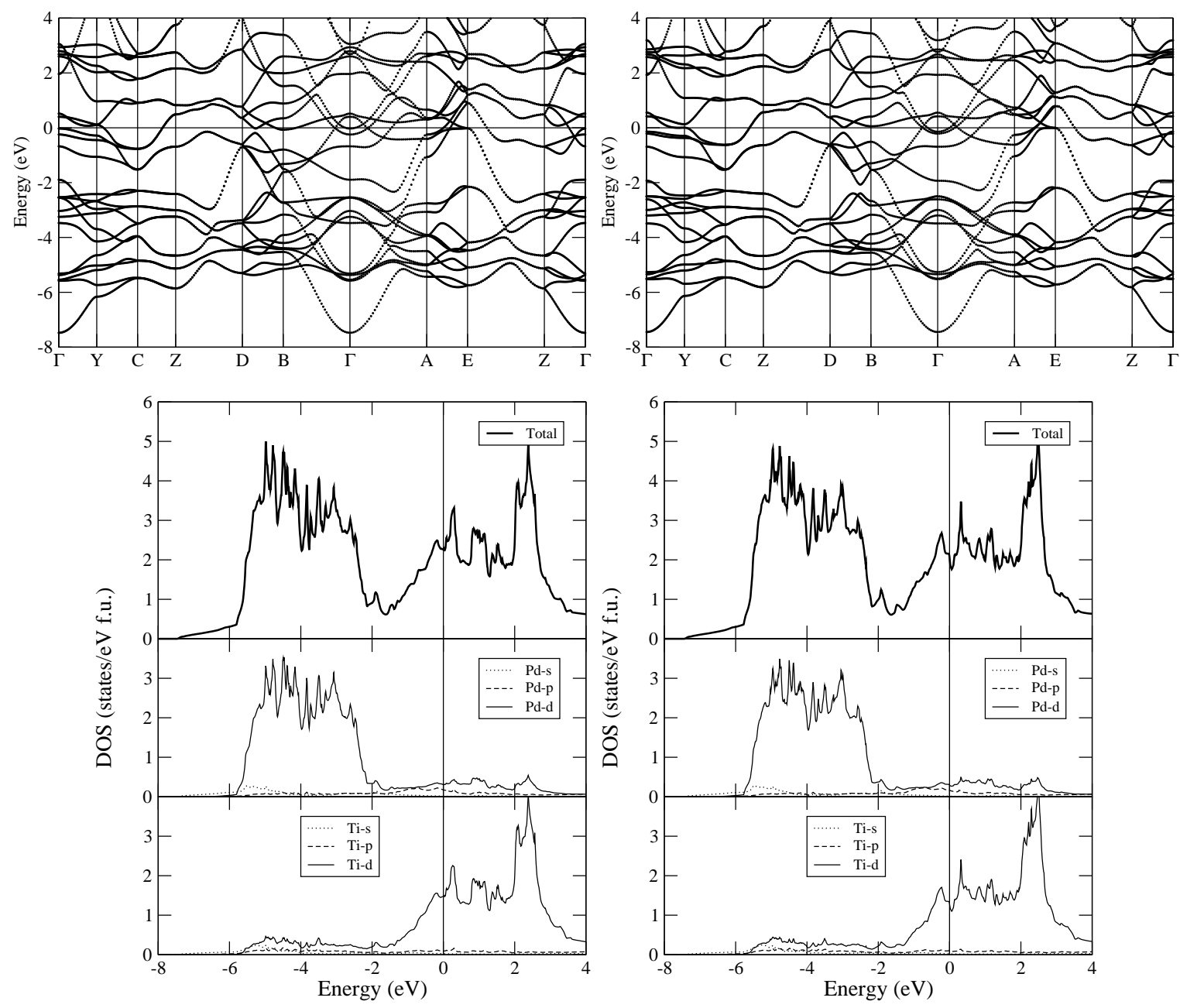

FIG. 4: Band structures, densities of states and partial densities of states of PdTi at relaxed B19 (left) and equilibrium $B 19^{\prime}$ (right) structures. Labelling of the $B 19$ band structure is as a special case of $B 19^{\prime}$.

$\cos \gamma=a / 2 b$ and $4 u-1=2 v$, orthorhombic symmetry is restored. The side of the conventional cell of this body centered orthorhombic (bco) (space group Pmna) structure is doubled in the $\mathbf{b}$ direction, though the primitive cell still contains four atoms. Although at relatively high energy ( II), this provides us a reference point for structures with large $\gamma \sqrt[37]{ }$. Note that a further shear to $\cos \gamma=a / b$ would give the $B 19$ structure once more.

The potential energies of continuous paths between the structures identified above are important for understanding the transformation mechanism. We compute the energies of three paths: $B 2-B 19, B 19-B 19^{\prime}$ and $B 19^{\prime}$-bco. In keeping with the timescale separation between bulk strain and atomic motion, we define intermediate configurations by relaxing the atoms to their minimum energy configuration consistent with the applied symmetry and strain on the cell. The remaining four strain degrees of freedom are reduced to a single parameter by taking interpolations between the strains of the endpoint structures. For the $B 2$ structure, we minimize the energy assuming the $B 19$ space group, which gives a smooth evo- lution of the structure along the path. ¿From Figure 3 , it is clear that there is no total energy barrier along the $B 2-B 19-B 19^{\prime}$ path, and that $B 19$ represents the total energy barrier between $B 19^{\prime}$ martensitic variants. The $B 19$ phase can be viewed as the binary equivalent of hcp, and the transformation path as the binary equivalent of the Nishiyama-Wassermann (NW) path. Using the analogy with the NW mechanism for the bcc-hcp transition, we can attribute the transition to a shuffling of $(110)_{b c c}$ planes 11 .

Structural instabilities in metals are typically related to details of the Fermi surface, and we have calculated the band structures of $B 2, B 19$ and $B 19^{\prime}$ to investigate this. In $\mathrm{PdTi} / \mathrm{PtTi}$ the band structure is dominated by the $d$-bands, with the $\mathrm{Pd} / \mathrm{Pt}$ bands lying below the Fermi level and almost fully occupied, and the Ti $d$-bands lying above the Fermi level (Figure 4 and 5), the band centers being offset by some $6 \mathrm{eV}$. The free-electron like $s p$ bands are very broad, and play little role in the bonding except to donate some electrons to the Ti-d band. The large strain involved in the $B 2-B 19$ transition means 

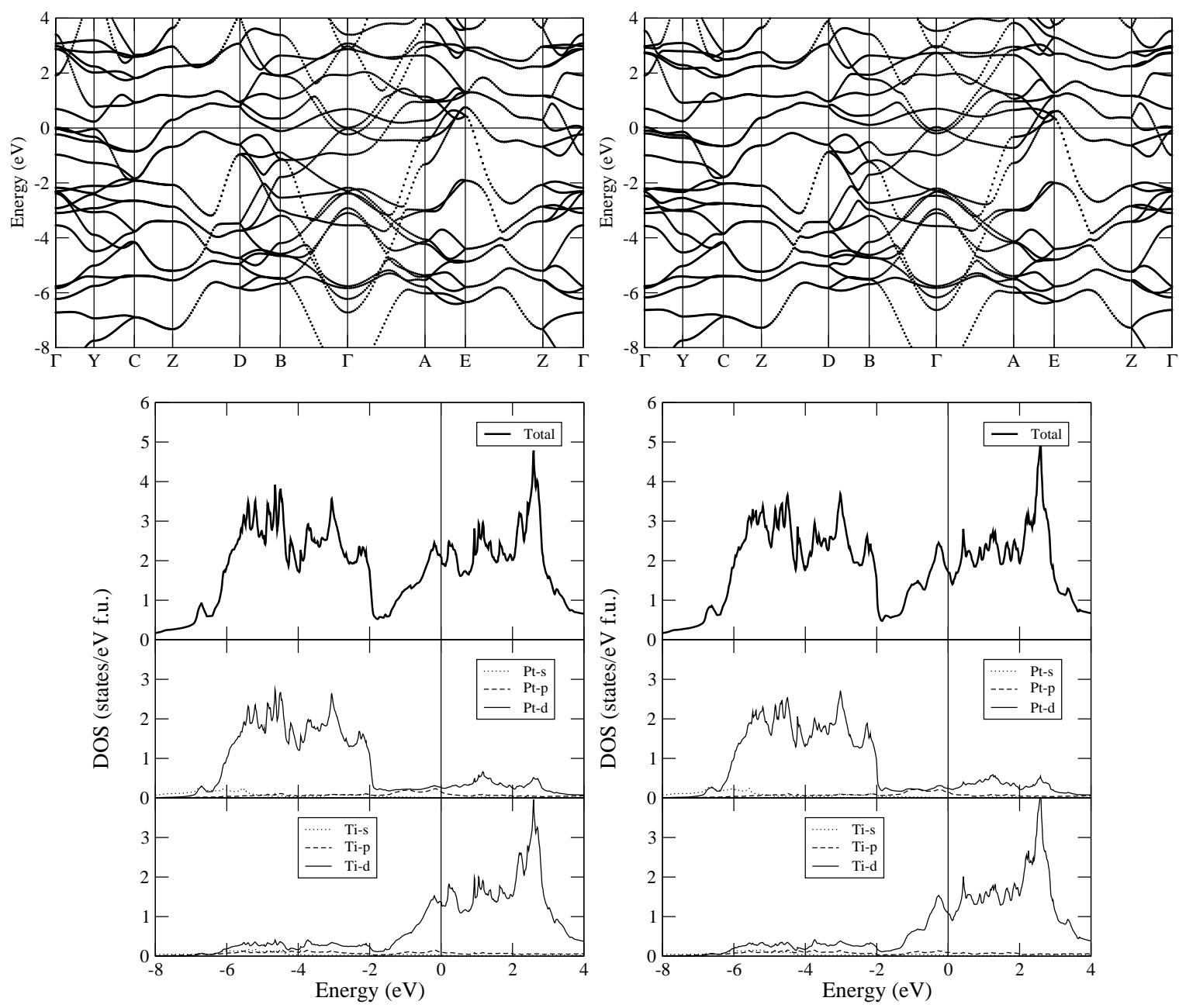

FIG. 5: Band structures, densities of states and partial densities of states of PtTi at relaxed B19 (left) and equilibrium $B 19^{\prime}$ (right) structures. Labelling of the $B 19$ band structure is as a special case of $B 19^{\prime}$.

that the Fermi surfaces are quite different ( $B 19$ having the lowest DOS at $E_{F} \sqrt{16}$ ) and this transition cannot be related directly to the band structure. By contrast, the $B 19-B 19^{\prime}$ transition is accompanied by the opening of a pseudogap at the Fermi level, a typical signature of increased stability. The band structures are very similar, the small difference which stabilises $B 19^{\prime}$ being traceable to the shifting above the Fermi level of a pocket of electrons around $B$. The distortion to $B 19^{\prime}$ is just enough to complete the topological phase transition which eliminates this pocket of electrons in both materials.

\section{CONCLUSIONS}

In conclusion, we have performed ab initio calculations of the structural energetics of PdTi and PtTi. In each case we predict that the low temperature ground state structure will be $B 19^{\prime}$, with the (observed) $B 2$ and $B 19$ phases being dynamically stabilized. There are no total energy barriers between the structures, meaning that the phase space microstates that belong to the $B 19^{\prime}$ structure also belong to the $B 19$ and $B 2$ structures.

In contrast to NiTi 10], the entire $\Gamma-M$ phonon branch is unstable. We showed that the $B 19$ structure can be obtained by a "freezing in" of phonons of the $B 2$ structure coupled to the shear associated with the $c^{\prime}=\left(c_{11}-c_{12}\right) / 2$ elastic constant, but that no single dynamical-matrix or force-constant-matrix eigenvector leads to the lowsymmetry phase.

The $B 19^{\prime}$ then corresponds to a further strain coupled to a $\Gamma_{4}$ phonon of the $B 19$ phase. Tracing the atomic motions of these instabilities shows that they are both related to shears of alternate $(110)_{B 2}$ phases, and hence that the transition mechanism is the binary equivalent of the Nishiyama-Wassermann bcc-hcp mechanism.

\section{Acknowledgments}

We thank R. D. James, I. I. Naumov, and K. Bhattacharya for valuable discussions. This work was sup- 
ported by AFOSR/MURI F49620-98-1-0433. The calculations were performed on the SGI Origin 3000 and IBM
SP3 at ARL MSRC.
[1] U. Pinsook and G. J. Ackland, Phys. Rev. B 58, 11252 (1998); Phys. Rev. B 625427 (2000).

[2] W. Cochran, Adv. Phys. 9, 387 (1960).

[3] G. J. Ackland and M. C. Warren, Phase Transitions, 61, 215 (1997).

[4] M. C. Warren and G. J. Ackland, Phys. Chem. Minerals 23, 107 (1996).

[5] B. B. Karki, M. C. Warren, L. Stixrude, G. J. Ackland and J. Crain, Phys. Rev. B 55, 3465 (1997).

[6] R. D. King-Smith and David Vanderbilt, Phys. Rev. B 49, 5828 (1994).

[7] R. Yu and H. Krakauer, Phys. Rev. Lett. 74, 4067 (1995).

[8] Ph. Ghosez, E. Cockayne, U. V. Waghmare and K. M. Rabe, Phys. Rev. B 60, 836 (1999).

[9] U. V. Waghmare and K. M. Rabe, Phys. Rev. B 55, 6161 (1997).

[10] X. Huang, C. Bungaro, V. Godlevsky and K. M. Rabe, Phys. Rev. B 65, 14108 (2002).

[11] B. Winn, S. M. Shapiro, D. Schlagel and T. Lograsso, unpublished).

[12] A. E. Dwight, R. A. Conner, Jr. and J. W. Downey, Acta Crystallogr. 18, 835 (1965).

[13] H. C. Donkersloot and J. H. N. van Vucht, J. LessCommon Met. 20, 83 (1970).

[14] G. Bihlmayer, R. Eibler and A. Neckel, Phys. Rev. B 50, 13113 (1994).

[15] G. Bihlmayer, R. Eibler and A. Neckel, Philos. Mag. B 73, 511 (1996).

[16] Y. Y. Ye, C. T. Chan and K. M. Ho, Phys. Rev. B 56, 3678 (1997).

[17] G. Kresse and J. Hafner, Phys. Rev. B 47, R558 (1993).

[18] G. Kresse and J. Furthmüller, Phys. Rev. B 54, 11169 (1996).

[19] J. P. Perdew and A. Zunger, Phys. Rev. B 23, 5048 (1981).

[20] D. Vanderbilt, Phys. Rev. B 41, 7892 (1990).

[21] M. Methfessel and A. T. Paxton, Phys. Rev. B 40, 3616 (1989).

[22] P. E. Blöchl, O. Jepsen and O. K. Andersen, Phys. Rev. B 49, 16223 (1994).

[23] S. Baroni, S. de. Gironcoli, A. Dal Corso and P. Gian- nozzi, http://www.sissa.it/cm/PWcodes,

[24] A. M. Rappe, K. M. Rabe, E. Kaxiras and J. D. Joannopoulos, Phys. Rev. B 41, 1227 (1990).

[25] A. DalCorso, A. Pasquarello and A. Baldereschi, Phys. Rev. B 56, 11369 (1997).

[26] P. Giannozzi, S. de Gironcoli, P. Pavone and S. Baroni, Phys. Rev. B 43, 7231 (1991).

[27] P. Blaha, K. Schwarz, and J. Luitz, WIEN97, Vienna University of Technology, Vienna 1997. (Improved and updated Unix version of the original copyrighted WIENcode, published by P. Blaha, K. Schwarz, P. Sorantin, and S. B. Trickey, in Comput. Phys. Commun. 59, 399 1990). Our FLAPW calculations were performed using a $120 k$-point mesh in the $\frac{1}{48}$ irreducible wedge. No shape approximations were made to the density or potential. The muffin-tin radii for $\mathrm{Pd} / \mathrm{Pt}$ and $\mathrm{Ti}$ were chosen to be 1.343Åand $1.278 \AA$, repectively. A $R_{M T} K_{M A X}$ of 10 were used. The tetrahedron method was used in the Brillouin zone integrations.

[28] F. Bassani and G. Pastori Parravicini, Electronic states and optical transitions in solids, ed. by R. A. Ballinger (Oxford, New York, Pergamon Press, 1975).

[29] M. Born and K. Huang, Dynamical Theory of Crystal Lattices, (Oxford University Press, Oxford, 1956).

[30] S. K. Sikka, Y. K. Vohra and R. Chidambaram, Prog. Mat. Sci. 27, 245 (1982).

[31] J. M. Ball and R. D. James, Zeitschrift fur Angewandte Mathematik und Mechanik S2 76, 389 (1996).

[32] A. A. Kelsey and G. J. Ackland, J. Phys. CM, 12, 32, (2000).

[33] Y. Kudoh, M. Tokonami, S. Miyazaki and K. Otsuka, Acta Metall. Mater. 33, 2049 (1985).

[34] G. J. Ackland, M. C. Warren and S. J. Clark, J. Phys. Cond. Mat. 9, 7861 (1997).

[35] W. G. Moffatt, The handbook of binary phase diagrams (Genium Publishing, Schenectady, New York, 1984).

[36] T. Biggs, M. B. Cortie, M. J. Witcomb and L. A. Cornish, Metall. Mater. Trans A 32A, 1881 (2001).

[37] According to various density functional calculations, this body centered orthorhombic structure appears to be more stable than $B 19^{\prime}$ in stoichiometric NiTi. 